\title{
GEOMETRY OF THE COMPLEX OF CURVES AND OF TEICHMÜLLER SPACE
}

\author{
URSULA HAMENSTÄDT
}

\begin{abstract}
Using train tracks on a non-exceptional oriented surface $S$ of finite type in a systematic way we give a proof that the complex of curves $\mathcal{C}(S)$ of $S$ is a hyperbolic geodesic metric space. We also discuss the relation between the geometry of the complex of curves and the geometry of Teichmüller space.
\end{abstract}

\section{INTRODUCTION}

Consider a compact oriented surface $S$ of genus $g \geq 0$ from which $m \geq 0$ points, so-called punctures, have been deleted. We assume that $3 g-3+m \geq 2$; this rules out a sphere with at most four punctures and a torus with at most one puncture.

In [10, Harvey associates to such a surface the following simplical complex.

Definition 1.1. The complex of curves $\mathcal{C}(S)$ for the surface $S$ is the simplicial complex whose vertices are free homotopy classes of essential simple closed curves on $S$ and whose simplices are spanned by collections of such curves which can be realized disjointly.

Here we mean by an essential simple closed curve a simple closed curve which is not contractible nor homotopic into a puncture. Since $3 g-3+m$ is the number of curves in a pants decomposition of $S$, i.e. a maximal collection of disjoint mutually not freely homotopic essential simple closed curves which decompose $S$ into $2 g-$ $2+m$ open subsurfaces homeomorphic to a thrice punctured sphere, the dimension of $\mathcal{C}(S)$ equals $3 g-3+m-1$.

In the sequel we restrict our attention to the one-skeleton of the complex of curves which we denote again by $\mathcal{C}(S)$ by abuse of notation. Since $3 g-3+m \geq 2$ by assumption, $\mathcal{C}(S)$ is a nontrivial graph which moreover is connected [10. However, this graph is locally infinite. Namely, for every simple closed curve $\alpha$ on $S$ the surface $S-\alpha$ which we obtain by cutting $S$ open along $\alpha$ contains at least one connected component of Euler characteristic at most -2, and such a component contains infinitely many distinct free homotopy classes of simple closed curves which viewed as curves in $S$ are disjoint from $\alpha$.

Providing each edge in $\mathcal{C}(S)$ with the standard euclidean metric of diameter 1 equips the complex of curves with the structure of a geodesic metric space. Since $\mathcal{C}(S)$ is not locally finite, this metric space $(\mathcal{C}(S), d)$ is not locally compact. Masur and Minsky [18] showed that nevertheless its geometry can be understood quite explicitly. Namely, $\mathcal{C}(S)$ is hyperbolic of infinite diameter. Here for some $\delta>0$ a geodesic metric space is called $\delta$-hyperbolic in the sense of Gromov if it satisfies the $\delta$-thin triangle condition: For every geodesic triangle with sides $a, b, c$ the side

Date: March 18, 2005.

Partially supported by Sonderforschungsbereich 611 . 
$c$ is contained in the $\delta$-neighborhood of $a \cup b$. Later Bowditch [2] gave a simplified proof of the result of Masur and Minsky which can also be used to compute explicit bounds for the hyperbolicity constant $\delta$.

Since the Euler characteristic of $S$ is negative, the surface $S$ admits a complete hyperbolic metric of finite volume. The group of diffeomorphisms of $S$ which are isotopic to the identity acts on the space of such metrics, and the quotient space under this action is the Teichmüller space $\mathcal{T}_{g, m}$ for $S$ of all marked isometry classes of complete hyperbolic metrics on $S$ of finite volume, or, equivalently, the space of all complex structures on $S$ of finite type. The Teichmüller space can be equipped with a natural topology, and with this topology it is homeomorphic to $\mathbb{R}^{6 g-6+2 m}$. The mapping class group $\mathcal{M}_{g, m}$ of all isotopy classes of orientation preserving diffeomorphisms of $S$ acts properly discontinuously as group of diffeomorphisms on Teichmüller space preserving a complete Finsler metric, the so-called Teichmüller metric. The quotient orbifold is the moduli space $\operatorname{Mod}(S)$ of $S$ of all isometry classes of complete hyperbolic metrics of finite volume on $S$ (for all this see [1]).

The significance of the complex of curves for the geometry of Teichmüller space comes from the obvious fact that the mapping class group acts on $\mathcal{C}(S)$ as a group of simplicial isometries. Even more is true: If $S$ is not a twice punctured torus or a closed surface of genus 2, then the extended mapping class group of all isotopy classes of all diffeomorphisms of $S$ coincides precisely with the group of simplicial isometries of $\mathcal{C}(S)$; for a closed surface of genus 2, the group of simplicial isometries of $\mathcal{C}(S)$ is the quotient of the extended mapping class group under the hyperelliptic involution which acts trivially on $\mathcal{C}(S)$ (see [12 for an overview on this and related results). Moreover, there is a natural map $\Psi: \mathcal{T}_{g, m} \rightarrow \mathcal{C}(S)$ which is coarsely $\mathcal{M}_{g, m}$-equivariant and coarsely Lipschitz with respect to the Teichmüller metric on

$\mathcal{T}_{g, m}$. By this we mean that there is a number $a>1$ such that $d(\Psi(\phi h), \phi(\Psi h)) \leq a$ for all $h \in \mathcal{T}_{g, m}$ and all $\phi \in \mathcal{M}_{g, m}$ and that moreover $d\left(\Psi h, \Psi h^{\prime}\right) \leq a d_{T}\left(h, h^{\prime}\right)+a$ for all $h, h^{\prime} \in \mathcal{T}_{g, m}$ where $d_{T}$ denotes the distance function on $\mathcal{T}_{g, m}$ induced by the Teichmüller metric.

As a consequence, the geometry of $\mathcal{C}(S)$ can be related to the large-scale properties of the geometry of Teichmüller space and the mapping class group. We discuss this relation in Section 4 of this note. In Section 3 we give a proof of hyperbolicity of the complex of curves using train tracks and splitting sequences of train tracks in a consistent way as the main tool. Section 2 contains a summary of those properties of train tracks and geodesic laminations which are needed in Section 3 and Section 4.

\section{TRAin tracks AND GEODESIC LAMINATIONS}

In this section we introduce train tracks and geodesic laminations for our surface $S$, and we discuss their most important properties.

To begin with, choose a complete hyperbolic metric on $S$ of finite volume. With respect to this metric, every essential free homotopy class can be represented by a closed geodesic which is unique up to parametrization. This geodesic is simple, i.e. without self-intersection, if and only if the free homotopy class has a simple representative (see 44). In other words, there is a 1-1-correspondence between points in the complex of curves and simple closed geodesics on $S$. Moreover, there is a fixed compact subset $S_{0}$ of $S$ containing all simple closed geodesics. 
The Hausdorff distance between two closed bounded subsets $A, B$ of a metric space $X$ is defined to be the infimum of all numbers $\epsilon>0$ such that $A$ is contained in the $\epsilon$-neighborhood of $B$ and $B$ is contained in the $\epsilon$-neighborhood of $A$. This defines indeed a distance and hence a topology on the space of closed bounded subsets of $X$; this topology is called the Hausdorff topology. If $X$ is compact then the space of closed subsets of $X$ is compact as well. In particular, for the distance on $S$ induced by a complete hyperbolic metric of finite volume, the space of closed subsets of the compact set $S_{0} \subset S$ is compact with respect to the Hausdorff topology.

The collection of all simple closed geodesics on $S$ is not a closed set with respect to the Hausdorff topology, but a point in its closure can be described as follows.

Definition 2.1. A geodesic lamination for a complete hyperbolic structure of finite volume on $S$ is a compact subset of $S$ which is foliated into simple geodesics.

Thus every simple closed geodesic is a geodesic lamination which consists of a single leaf. The space of geodesic laminations on $S$ equipped with the Hausdorff topology is compact, and it contains the closure of the set of simple closed geodesics as a proper subset. Note that every lamination in this closure is necessarily connected.

To describe the structure of the space of geodesic laminations more explicitly we introduce some more terminology.

Definition 2.2. A geodesic lamination $\lambda$ is called minimal if each of its halfleaves is dense in $\lambda$. A geodesic lamination $\lambda$ is maximal if all its complementary components are ideal triangles or once punctured monogons. A geodesic lamination is called complete if it is maximal and can be approximated in the Hausdorff topology for compact subsets of $S$ by simple closed geodesics.

As an example, a simple closed geodesic is a minimal geodesic lamination. A minimal geodesic lamination with more than one leaf has uncountably many leaves. Every minimal geodesic lamination can be approximated in the Hausdorff topology by simple closed geodesics [5]. Moreover, a minimal geodesic lamination $\lambda$ is a sublamination of a complete geodesic lamination [7, i.e. there is a complete geodesic lamination which contains $\lambda$ as a closed subset. In particular, every simple closed geodesic on $S$ is a sublamination of a complete geodesic lamination. Every geodesic lamination $\lambda$ is a disjoint union of finitely many minimal components and a finite number of isolated leaves. Each of the isolated leaves of $\lambda$ either is an isolated closed geodesic and hence a minimal component, or it spirals about one or two minimal components [1, 5, 22]. This means that the set of accumulation points of an isolated half-leaf of $\lambda$ is a minimal component of $\lambda$.

Geodesic laminations which are disjoint unions of minimal components can be equipped with the following additional structure.

Definition 2.3. A measured geodesic lamination is a geodesic lamination together with a translation invariant transverse measure.

A transverse measure for a geodesic lamination $\lambda$ assigns to every smooth compact arc $c$ on $S$ with endpoints in the complement of $\lambda$ and which intersects $\lambda$ transversely a finite Borel measure on $c$ supported in $c \cap \lambda$. These measures transform in the natural way under homotopies of $c$ by smooth arcs transverse to $\lambda$ which move the endpoints of the $\operatorname{arc} c$ within fixed complementary components. The support of the measure is the smallest sublamination $\nu$ of $\lambda$ such that the measure on 
any $\operatorname{arc} c$ which does not intersect $\nu$ is trivial. This support is necessarily a union of minimal components of $\lambda$. An example for a measured geodesic lamination is a weighted simple closed geodesic which consists of a simple closed geodesic $\alpha$ and a positive weight $a>0$. The measure disposed on a transverse $\operatorname{arc} c$ is then the sum of the Dirac masses on the intersection points between $c$ and $\alpha$ multiplied with the weight $a$.

The space $\mathcal{M L}$ of measured geodesic laminations on $S$ can naturally be equipped with the weak $k^{*}$-topology. This topology restricts to the weak*-topology on the space of measures on a given arc $c$ which is transverse to each lamination from an open subset of lamination space. The natural action of the group $(0, \infty)$ by scaling is continuous with respect to this topology, and the quotient is the space $\mathcal{P} \mathcal{M L}$ of projective measured geodesic laminations. This space is homeomorphic to a sphere of dimension $6 g-7+2 m$ (see [5, 6, 23]).

Measured geodesic laminations are intimately related to more classical objects associated to Riemann surfaces, namely holomorphic quadratic differentials . A holomorphic quadratic differential $q$ on a Riemann surface $S$ assigns to each complex coordinate $z$ an expression of the form $q(z) d z^{2}$ where $q(z)$ is a holomorphic function on the domain of the coordinate system, and $q(z)(d z / d w)^{2}=q(w)$ for overlapping coordinates $z, w$. We require that $q$ has at most a simple pole at each puncture of $S$. If $q$ does not vanish identically, then its zeros are isolated and independent of the choice of a complex coordinate. If $p$ is not a zero for $q$ then there is a coordinate $z$ near $p$, unique up to multiplication with \pm 1 , such that $p$ corresponds to the origin and that $q(z) \equiv 1$. Writing $z=x+i y$ for this coordinate, the euclidean metric $d x^{2}+d y^{2}$ is uniquely determined by $q$. The arcs parallel to the $x$-axis (or $y$-axis, respectively) define a foliation $\mathcal{F}_{h}$ (or $\mathcal{F}_{v}$ ) on the set of regular points of $q$ called the horizontal (or vertical) foliation. The vertical length $|d y|$ defines a transverse measure for the horizontal foliation, and the horizontal length $|d x|$ defines a transverse measure for the vertical foliation. The foliations $\mathcal{F}_{h}, \mathcal{F}_{v}$ have singularities of the same type at the zeros of $q$ and at the punctures of $S$ (see 25 for more on quadratic differentials and measured foliations).

There is a one-one-correspondence between measured geodesic laminations and (equivalence classes of) measured foliations on $S$ (see [15] for a precise statement). The pair of measured foliations defined by a quadratic differential $q$ corresponds under this identification to a pair of measured geodesic laminations $\lambda \neq \mu \in \mathcal{M L}$ which jointly fill up $S$. This means that every simple closed geodesic intersects the support of either $\lambda$ or $\mu$ transversely. Vice versa, every pair of measured geodesic laminations $\lambda \neq \mu \in \mathcal{M L}$ which jointly fill up $S$ defines a unique complex structure of finite type on $S$ together with a holomorphic quadratic differential (see [13] and the references given there).

Thurston invented a way to understand the structure of the space of geodesic laminations by squeezing almost parallel strands of such a lamination to a simple arc and analyzing the resulting graph. The structure of such a graph is as follows.

Definition 2.4. A train track on the surface $S$ is an embedded 1-complex $\tau \subset S$ whose edges (called branches) are smooth arcs with well-defined tangent vectors at the endpoints. At any vertex (called a switch) the incident edges are mutually tangent. Through each switch there is a path of class $C^{1}$ which is embedded in $\tau$ and contains the switch in its interior. In particular, the branches which are incident on a fixed switch are divided into "incoming" and "outgoing" branches according 
to their inward pointing tangent at the switch. Each closed curve component of $\tau$ has a unique bivalent switch, and all other switches are at least trivalent. The complementary regions of the train track have negative Euler characteristic, which means that they are different from discs with 0,1 or 2 cusps at the boundary and different from annuli and once-punctured discs with no cusps at the boundary. A train track is called generic if all switches are at most trivalent.

In the sequel we only consider generic train tracks. For such a train track, every complementary component is a bordered subsurface of $S$ whose boundary consists of a finite number of arcs of class $C^{1}$ which come together at a finite number of cusps. Moreover, for every switch of $\tau$ there is precisely one complementary component containing the switch in its closure which has a cusp at the switch. A detailed account on train tracks can be found in 23] and 21].

A geodesic lamination or a train track $\lambda$ is carried by a train track $\tau$ if there is a map $F: S \rightarrow S$ of class $C^{1}$ which is isotopic to the identity and maps $\lambda$ to $\tau$ in such a way that the restriction of its differential $d F$ to every tangent line of $\lambda$ is non-singular. Note that this makes sense since a train track has a tangent line everywhere.

If $c$ is a simple closed curve carried by $\tau$ with carrying map $F: c \rightarrow \tau$ then $c$ defines a counting measure $\mu_{c}$ on $\tau$. This counting measure is the non-negative weight function on the branches of $\tau$ which associates to an open branch $b$ of $\tau$ the number of connected components of $F^{-1}(b)$. A counting measure is an example for a transverse measure on $\tau$ which is defined to be a nonnegative weight function $\mu$ on the branches of $\tau$ satisfying the switch condition: For every switch $s$ of $\tau$, the sum of the weights over all incoming branches at $s$ is required to coincide with the sum of the weights over all outgoing branches at $s$. The set $V(\tau)$ of all transverse measures on $\tau$ is a closed convex cone in a linear space and hence topologically it is a closed cell. More generally, every measured geodesic lamination $\lambda$ on $S$ which is carried by $\tau$ via a carrying map $F: \lambda \rightarrow \tau$ defines a transverse measure on $\tau$ by assigning to a branch $b$ the total mass of the pre-image of $b$ under $F$; the resulting weight function is independent of the particular choice of $F$. Moreover, every transverse measure for $\tau$ can be obtained in this way (see [23]).

Definition 2.5. A train track is called recurrent if it admits a transverse measure which is positive on every branch. A train track $\tau$ is called transversely recurrent if every branch $b$ of $\tau$ is intersected by an embedded simple closed curve $c=c(b) \subset S$ which intersects $\tau$ transversely and is such that $S-\tau-c$ does not contain an embedded bigon, i.e. a disc with two corners at the boundary. A recurrent and transversely recurrent train track is called birecurrent. A generic train track which carries a complete geodesic lamination is called complete.

For every recurrent train track $\tau$, measures which are positive on every branch define the interior of the convex cone $V(\tau)$ of all transverse measures. A complete train track is birecurrent [7.

A half-branch $\tilde{b}$ in a generic train track $\tau$ incident on a switch $v$ is called large if the switch $v$ is trivalent and if every $\operatorname{arc} \rho:(-\epsilon, \epsilon) \rightarrow \tau$ of class $C^{1}$ which passes through $v$ meets the interior of $\tilde{b}$. A branch $b$ in $\tau$ is called large if each of its two half-branches is large; in this case $b$ is necessarily incident on two distinct switches (for all this, see [23]). 
There is a simple way to modify a transversely recurrent train track $\tau$ to another transversely recurrent train track. Namely, if $e$ is a large branch of $\tau$ then we can perform a right or left split of $\tau$ at $e$ as shown in Figure A below. The split $\tau^{\prime}$ of a train track $\tau$ is carried by $\tau$. If $\tau$ is complete and if the complete geodesic lamination $\lambda$ is carried by $\tau$, then for every large branch $e$ of $\tau$ there is a unique choice of a right or left split of $\tau$ at $e$ with the property that the split track $\tau^{\prime}$ carries $\lambda$, and $\tau^{\prime}$ is complete. In particular, a complete train track $\tau$ can always be split at any large branch $e$ to a complete train track $\tau^{\prime}$; however there may be a choice of a right or left split at $e$ such that the resulting train track is not complete any more (compare p.120 in [23]).

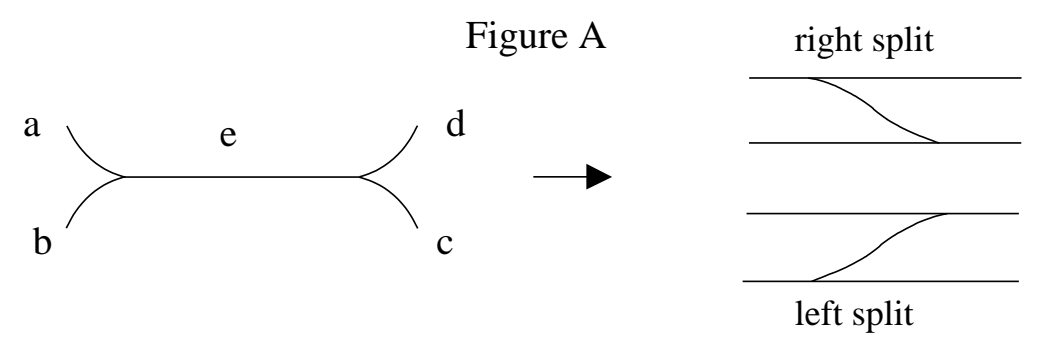

In the sequel we denote by $\mathcal{T} T$ the collection of all isotopy classes of complete train tracks on $S$. A sequence $\left(\tau_{i}\right)_{i} \subset \mathcal{T} T$ of complete train tracks is called a splitting sequence if $\tau_{i+1}$ can be obtained from $\tau_{i}$ by a single split at some large branch $e$.

\section{Hyperbolicity of the COMPlex of CURVES}

In this section we present a proof of hyperbolicity of the complex of curves. Our main strategy coincides with the approach used by Masur and Minsky [18] and Bowditch 2. Namely, the first step consists in guessing a family of uniform quasi-geodesics in the complex of curves connecting any two points. Here a $q$-quasigeodesic for some $q>1$ is a curve $c:[a, b] \rightarrow \mathcal{C}(S)$ which satisfies

$$
d(c(s), c(t)) / q-q \leq|s-t| \leq q d(c(s), c(t))+q \text { for all } s, t \in[a, b] .
$$

Note that a quasi-geodesic does not have to be continuous. In a hyperbolic geodesic metric space, every $q$-quasi-geodesic is contained in a fixed tubular neighborhood of any geodesic joining the same endpoints, so the $\delta$-thin triangle condition also holds for triangles whose sides are uniform quasi-geodesics [3]. As a consequence, for every triangle in a hyperbolic geodesic metric space with uniform quasi-geodesic sides there is a "midpoint" whose distance to each side of the triangle is bounded from above by a universal constant. The second step of the proof consists in finding such a midpoint for triangles whose sides are curves of the distinguished curve family. This is then used in a third step to establish the $\delta$-thin triangle condition for the distinguished family of curves and derive from this hyperbolicity of $\mathcal{C}(S)$. By abuse of notation, in the sequel we simply write $\alpha \in \mathcal{C}(S)$ if $\alpha$ is a free homotopy class of an essential simple closed curve on $S$, i.e. if $\alpha$ is a vertex of $\mathcal{C}(S)$.

We begin with defining a map from the set $\mathcal{T} T$ of complete train tracks on $S$ into $\mathcal{C}(S)$. For this we call a transverse measure $\mu$ for a complete train track $\tau$ a vertex cycle [18] if $\mu$ spans an extreme ray in the convex cone $V(\tau)$ of all transverse measures on $\tau$. 
Up to scaling, every vertex cycle $\mu$ is a counting measure of a simple closed curve $c$ which is carried by $\tau$ [18. Namely, the switch conditions are a family of linear equations with integer coefficients for the transverse measures on $\tau$. Thus an extreme ray is spanned by a nonnegative rational solution which can be scaled to a nonnegative integral solution. Define a simple multi-curve on $S$ to be a collection $c=c_{1} \cup \cdots \cup c_{\ell} \subset \mathcal{C}(S)$ of free homotopy classes of simple closed curves which can be realized disjointly. If in addition there is a positive weight $a_{i}>0$ associated to each of these curves then we speak of a simple weighted multi-curve. Note that there is a natural 1-1-correspondence between simple weighted multi-curves and measured geodesic laminations supported on simple closed geodesics. From every integral transverse measure $\mu$ for $\tau$ we can construct a unique simple weighted multi-curve carried by $\tau$ whose counting measure coincides with $\mu$ as follows. For each branch $b$ of $\tau$ draw $\mu(b)$ disjoint arcs parallel to $b$. By the switch condition, the endpoints of these arcs can be connected near the switches in a unique way so that the resulting family of arcs does not have self-intersections. Let $c$ be the simple multicurve consisting of the free homotopy classes of the connected components of the resulting curve $\tilde{c}$. To each such homotopy class associate the number of components of $\tilde{c}$ in this class as a weight. The resulting simple weighted multi-curve is carried by $\tau$, and its counting measure equals $\mu$. Thus if there are two components of $\tilde{c}$ which are not freely homotopic then the weighted counting measures of these components determine a decomposition of $\mu$ into transverse measures for $\tau$ which are not multiples of $\mu$. This is impossible by assumption. Hence $c$ consists of a single class, and up to scaling, $\mu$ is the counting measure of a simple closed curve on $S$.

More precisely, a simple closed curve which is carried by $\tau$, with carrying map $F: c \rightarrow \tau$, defines a vertex cycle for $\tau$ if and only if $F(c)$ passes through every branch of $\tau$ at most twice, with different orientation (Lemma 2.2 of [8]). In particular, if $c$ is a vertex cycle for $\tau$ then its counting measure $\mu_{c}$ satisfies $\mu_{c}(b) \leq 2$ for every branch $b$ of $\tau$.

In the sequel we mean by a vertex cycle of a birecurrent train track $\tau$ an integral transverse measure on $\tau$ which is the counting measure of a simple closed curve $c$ on $S$ carried by $\tau$ and which spans an extreme ray of $V(\tau)$; we also use the notion vertex cycle for the simple closed curve $c$. Since the number of branches of a complete train track on $S$ only depends on the topological type of $S$, the number of vertex cycles for a complete train track on $S$ is bounded by a universal constant (see [18] and [8]).

The following observation of Penner and Harer 23] is essential for all what follows. Denote by $\mathcal{M C}(S)$ be the space of all simple multi-curves on $S$. Let $P=\cup_{i=1}^{3 g-3+m} \gamma_{i} \in \mathcal{M C}(S)$ be a pants decomposition for $S$, i.e. a simple multicurve with the maximal number of components. Then there is a special family of complete train tracks with the property that each pants curve $\gamma_{i}$ admits a closed neighborhood $A$ diffeomorphic to an annulus and such that $\tau \cap A$ is diffeomorphic to a standard twist connector depicted in Figure B. Such a train track clearly carries each pants curve from the pants decomposition $P$ as a vertex cycle; we call it adapted to $P$. For every complete geodesic lamination $\lambda$ there is a train track $\tau$ adapted to $P$ which carries $\lambda$ ([23, see also [7, [8]).

Since every simple multi-curve is a subset of a pants decomposition of $S$, we can conclude. 


\section{Figure B}
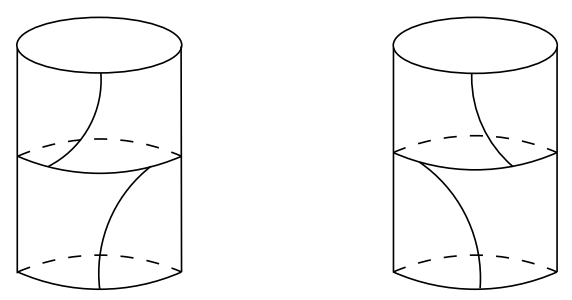

Lemma $3.1([7])$. For every pair $(\alpha, \beta) \in \mathcal{M C}(S) \times \mathcal{M C}(S)$ there is a splitting sequence $\left(\tau_{i}\right)_{0 \leq i \leq m} \subset \mathcal{T} T$ of complete train tracks with the property that each component of $\alpha$ is a vertex cycle for $\tau_{0}$ and that each component of $\beta$ is a vertex cycle for $\tau_{m}$.

We call a splitting sequence as in the lemma an $\alpha \rightarrow \beta$-splitting sequence. Note that such a sequence is by no means unique.

The intersection number $i(\gamma, \delta)$ between two simple closed curves $\gamma, \delta \in \mathcal{C}(S)$ equals the minimal number of intersection points between representatives of the free homotopy classes of $\gamma, \delta$. This intersection number extends bilinearly to a pairing for weighted simple multi-curves on $S$. The distance in $\mathcal{C}(S)$ between two simple closed curves $\alpha, \beta$ is bounded from above by $i(\alpha, \beta)+1$ (Lemma 1.1 of [2] and Lemma 2.1 of [18]). In particular, there is a number $D_{0}>0$ with the following property. Let $\tau, \tau^{\prime} \in \mathcal{T} T$ and assume that $\tau^{\prime}$ is obtained from $\tau$ by at most one split. Then the distance in $\mathcal{C}(S)$ between any vertex cycle of $\tau$ and any vertex cycle of $\tau^{\prime}$ is at most $D_{0}$ (see [18 and the discussion following Corollary 2.3 in [8]).

Define a map $\Phi: \mathcal{T} T \rightarrow \mathcal{C}(S)$ by assigning to a train track $\tau \in \mathcal{T} T$ a vertex cycle $\Phi(\tau)$ for $\tau$. By our above discussion, for any two choices $\Phi, \Phi^{\prime}$ of such a map we have $d\left(\Phi(\tau), \Phi^{\prime}(\tau)\right) \leq D_{0}$ for all $\tau \in \mathcal{T} T$. Images under the map $\Phi$ of splitting sequences then define a family of curves in $\mathcal{C}(S)$ which connect any pair of points in a $D_{0}$-dense subset of $\mathcal{C}(S) \times \mathcal{C}(S)$, equipped with the product metric. As a consequence, we can use such images of splitting sequences as our guesses for uniform quasi-geodesics. It turns our that up to parametrization, these curves are indeed $q$-quasi-geodesics in $\mathcal{C}(S)$ for a universal number $q>0$ only depending on the topological type of the surface $S$ ([19], see also []).

To explain this fact we use the following construction of Bowditch [2]. For multicurves $\alpha, \beta \in \mathcal{M C}(S)$ with $i(\alpha, \beta)>0$ and for numbers $a>0, r>0$ define

$$
L_{a}(\alpha, \beta, r)=\{\gamma \in \mathcal{C}(S) \mid \max \{a i(\gamma, \alpha), i(\gamma, \beta) / a i(\alpha, \beta)\} \leq r\} .
$$

Note that we have $L_{a}(\alpha, \beta, r)=L_{1 / a i(\alpha, \beta)}(\beta, \alpha, r)$ for all $r>0$, moreover $\alpha^{\prime} \in$ $L_{a}(\alpha, \beta, r)$ for every component $\alpha^{\prime}$ of $\alpha$ and every sufficiently large $a>0$, and $\beta^{\prime} \in L_{a}(\alpha, \beta, r)$ for every component $\beta^{\prime}$ of $\beta$ and every sufficiently small $a>0$. Thus for fixed $r>0$ we can think of a suitably chosen assignment which associates to a number $s>0$ a point in $L_{s}(\alpha, \beta, r)$ as a curve in $\mathcal{C}(S)$ connecting a component of $\beta$ to a component of $\alpha$ (provided, of course, that the sets $L_{s}(\alpha, \beta, r)$ are nonempty). Lemma 2.5 of [8] links such curves to splitting sequences.

Lemma $3.2([8])$. There is a number $k_{0} \geq 1$ with the following property. Let $\tau_{0} \in \mathcal{T} T$ be adapted to a pants decomposition $P$ of $S$, let $\left(\tau_{i}\right)_{0 \leq i \leq m} \subset \mathcal{T} T$ be a splitting sequence issuing from $\tau_{0}$ and let $\alpha \in \mathcal{M C}(S)$ be a multi-curve consisting of 
vertex cycles for $\tau_{m}$. Then there is a monotonous surjective function $\kappa:(0, \infty) \rightarrow$ $\{0, \ldots, m\}$ such that $\kappa(s)=0$ for all sufficiently small $s>0, \kappa(s)=m$ for all sufficiently large $s>0$ and that for all $s \in(0, \infty)$ there is a vertex cycle of $\tau_{\kappa(s)}$ which is contained in $L_{s}\left(\alpha, P, k_{0}\right)$.

Since for every multi-curve $\alpha \in \mathcal{M C}(S)$ and every pants decomposition $P$ of $S$ there is a $P \rightarrow \alpha$-splitting sequence, we conclude that for every $k \geq k_{0}$ and every $s>0$ the set $L_{s}(\alpha, P, k)$ is non-empty. To obtain a control of the size of these sets, Bowditch [2] uses the singular euclidean metrics on the surface $S$ defined the quadratic differentials determined by the multi-curves $\alpha$ and $P$. Namely, any two curves $\alpha, \beta \in \mathcal{C}(S)$ with $d(\alpha, \beta) \geq 3$ fill up $S$, i.e. they cut $S$ into components which are homeomorphic to discs and once punctured discs. Given two multicurves $\alpha, \beta \in \mathcal{M C}(S)$ with $d\left(\alpha^{\prime}, \beta^{\prime}\right) \geq 3$ for a component $\alpha^{\prime}$ of $\alpha, \beta^{\prime}$ of $\beta$, choose smooth representatives of $\alpha, \beta$, again denoted by $\alpha, \beta$, which intersect transversely in precisely $i(\alpha, \beta)$ points; for example, the geodesic representatives of $\alpha, \beta$ with respect to any complete hyperbolic metric on $S$ of finite volume have this property. We can now construct a piecewise euclidean metric on $S$ as follows. For each intersection point between $\alpha, \beta$ choose a closed rectangle in $S$ with piecewise smooth boundary containing this point in its interior and which does not contain any other intersection point between $\alpha, \beta$. We allow that some of the vertices of such a rectangle are punctures of $S$. These rectangles can be chosen in such a way that they provide $S$ with the structure of a cubical complex: The boundary of each component $D$ of $S-\alpha-\beta$ is a polygon with an even number of sides which are subarcs of $\alpha, \beta$ in alternating order. If $D$ does not contain a puncture, then its boundary has at least four sides. Thus we can construct the rectangles in such a way that their union is all of $S$ and that the intersection between any two distinct such rectangles either is a common side or a common vertex. Each rectangle from this cubical complex has two sides which are "parallel" to $\alpha$ and two sides "parallel" to $\beta$ (see Section 4 of [13] for a detailed discussion of this construction).

Let $a>0$ and write $b=1 / a i(\alpha, \beta)$. Equip each rectangle with an euclidean metric such that the sides parallel to $\alpha$ are of length $b$, the sides parallel to $\beta$ are of length $a$ and such that the metrics on two rectangles coincide on a common boundary arc. These metrics define a piecewise euclidean metric on $S$ with a singularity of cone angle $k \pi \geq 3 \pi$ in the interior of each disc component of $S-\alpha-\beta$ whose boundary consists of $2 k \geq 6$ sides. The metric also has a singularity of cone angle $\pi$ at each puncture of $S$ which is contained in a punctured disc component with two sides. Since there are precisely $i(\alpha, \beta)$ rectangles, the area of this piecewise euclidean metric on $S$ equals 1 .

The line segments of this singular euclidean metric which are parallel to $\alpha$ and $\beta$ define singular foliations $\mathcal{F}_{\alpha}, \mathcal{F}_{\beta}$ on $S$ with transverse measures induced by the singular metric. There is a holomorphic quadratic differential $q(a \alpha, b \beta)$ of area one for the complex structure on $S$ determined by the singular metric whose horizontal and vertical foliations are just $\mathcal{F}_{\alpha}, \mathcal{F}_{\beta}$, with transverse measure determined by the weight $a$. In other words, this structure is uniquely determined by the measured laminations $a \alpha, b \beta$. The assignment which associates to $a>0$ the Riemann surface structure determined by $q(a \beta, \beta / a i(\alpha, \beta))$ is up to parametrization just the geodesic in the Teichmüller space with respect to the Teichmüller metric defined by any one of the differentials $q(a \beta, \beta / i(\alpha, \beta))$ (compare [11, 13]). 
The following lemma is the main observation of Bowditch (Lemma 4.1 in [2]); its first part was earlier shown by Masur and Minsky (Lemma 5.1 of [18]).

Lemma 3.3 (2]). There is a number $k_{1} \geq k_{0}$ with the following property. For all $\alpha, \beta \in \mathcal{M C}(S)$ and every $a \in(0, \infty)$ there is some $\delta \in L_{a}\left(\alpha, \beta, k_{1}\right)$ such that for every $\gamma \in \mathcal{M C}(S)$ we have $i(\delta, \gamma) \leq k_{1} \max \{$ ai $(\alpha, \gamma), i(\gamma, \beta) / a i(\alpha, \beta)\}$. In particular, for every $R>0$, for all $\alpha, \beta \in \mathcal{M C}(S)$ and for every $a>0$ the diameter of the set $L_{a}(\alpha, \beta, R)$ is not bigger than $2 k_{1} R+1$.

Proof. In [18, 2] it is shown that there is a number $\nu>0$ only depending on the topological type of $S$ and there is an embedded essential annulus in $S$ whose width with respect to the piecewise euclidean metric is at least $\nu$, i.e. such that the distance between the boundary circles of the annulus is at least $\nu$. Assuming the existence of such an annulus, let $\delta$ be its core-curve. Then for every simple closed curve $\gamma$ on $S$ and for every essential intersection of $\gamma$ with $\delta$ there is a subarc of $\gamma$ which crosses through this annulus and hence whose length is at least $\nu$; moreover, different subarcs of $\gamma$ corresponding to different essential intersections between $\gamma$ and $\delta$ are disjoint. Thus the length with respect to the singular euclidean metric on $S$ of any simple closed curve $\gamma$ on $S$ is at least $\nu i(\gamma, \delta)$. On the other hand, by construction the minimal length with respect to this metric of a curve in the free homotopy class of $\gamma$ is bounded from above by $2 \max \{a i(\alpha, \gamma), i(\beta, \gamma) / a i(\alpha, \beta)\}$ and therefore the core curve $\delta$ of the annulus has the properties stated in the first part of our lemma (see [2]).

The second part of the lemma is immediate from the first. Namely, let $\alpha, \beta \in$ $\mathcal{M C}(S)$ and let $a>0$. Choose $\delta \in L_{a}\left(\alpha, \beta, k_{1}\right)$ which satisfies the properties stated in the first part of the lemma. If $\gamma \in L_{a}(\alpha, \beta, R)$ for some $R>0$ then we have $i(\gamma, \delta) \leq k_{1} R$ and hence $d(\gamma, \delta) \leq k_{1} R+1$.

As an immediate consequence of Lemma 3.2 and Lemma 3.3 we observe that there is a universal number $D_{1}>0$ with the following property. Let $P$ be a pants decomposition for $S$, let $\beta \in \mathcal{M C}(S)$ and let $\left(\tau_{i}\right)_{0 \leq i \leq m} \subset \mathcal{T} T$ be any $P \rightarrow \beta$ splitting sequence; then the Hausdorff distance in $\mathcal{C}(S)$ between the sets $\left\{\Phi\left(\tau_{i}\right) \mid 0 \leq\right.$ $i \leq m\}$ and $\cup_{a>0} L_{a}\left(\beta, P, k_{1}\right)$ is at most $D_{1} / 16$. In particular, for every $\beta \in \mathcal{C}(S)$ and every simple multi-curve $Q$ containing $\beta$ as a component the Hausdorff-distance between the sets $\cup_{a>0} L_{a}\left(\beta, P, k_{1}\right)$ and $\cup_{a>0} L_{a}\left(Q, P, k_{1}\right)$ is at most $D_{1} / 8$. Thus if $Q, Q^{\prime}$ are pants decompositions for $S$ containing a common curve $\beta \in \mathcal{C}(S)$ then the Hausdorff distance between $\cup_{a} L_{a}\left(Q, P, k_{1}\right)$ and $\cup_{a} L_{a}\left(Q^{\prime}, P, k_{1}\right)$ is at most $D_{1} / 4$.

On the other hand, for multi-curves $P, Q \in \mathcal{M C}(S)$ we have

$$
\cup_{a>0} L_{a}\left(P, Q, k_{1}\right)=\cup_{a>0} L_{a}\left(Q, P, k_{1}\right) .
$$

Therefore from two applications of our above consideration we obtain the following. Let $\alpha, \beta \in \mathcal{C}(S)$ and let $P, P^{\prime}, Q, Q^{\prime}$ be any pants decompositions for $S$ containing $\alpha, \beta$; then the Hausdorff distance between $\cup_{a>0} L_{a}\left(P, Q, k_{1}\right)$ and $\cup_{a>0} L_{a}\left(P^{\prime}, Q^{\prime}, k_{1}\right)$ is not bigger than $D_{1} / 2$. By our choice of $D_{1}$ this implies that the Hausdorff distance between the images under $\Phi$ of any $\alpha \rightarrow \beta$ - or $\beta \rightarrow \alpha$-splitting sequences is bounded from above by $D_{1}$.

Now let $\alpha, \beta, \gamma \in \mathcal{C}(S)$ be such that their pairwise distance in $\mathcal{C}(S)$ is at least 2 . Choose pants decompositions $P_{\alpha}, P_{\beta}, P_{\gamma}$ containing $\alpha, \beta, \gamma$. Then there are unique numbers $a, b, c>0$ such that $a b i\left(P_{\alpha}, P_{\beta}\right)=b c i\left(P_{\beta}, P_{\gamma}\right)=\operatorname{aci}\left(P_{\gamma}, P_{\alpha}\right)=1$. By 
construction, we have

$$
L_{a}\left(P_{\alpha}, P_{\beta}, k_{1}\right)=L_{b}\left(P_{\beta}, P_{\alpha}, k_{1}\right), L_{b}\left(P_{\beta}, P_{\gamma}, k_{1}\right)=L_{c}\left(P_{\gamma}, P_{\beta}, k_{1}\right)
$$

and $L_{c}\left(P_{\gamma}, P_{\alpha}, k_{1}\right)=L_{a}\left(P_{\alpha}, P_{\gamma}, k_{1}\right)$. Choose a point $\delta \in L_{a}\left(P_{\alpha}, P_{\beta}, k_{1}\right)$ such that for every $\zeta$ in $\mathcal{M C}(S)$ we have

$$
i(\delta, \zeta) \leq k_{1} \max \left\{\operatorname{ai}\left(P_{\alpha}, \zeta\right), i\left(\zeta, P_{\beta}\right) / \operatorname{ai}\left(P_{\alpha}, P_{\beta}\right)\right\}
$$

such a point exists by Lemma 3.3. Applying this inequality to $\zeta=P_{\gamma}$ yields $c i\left(\delta, P_{\gamma}\right) \leq k_{1}$. For $\zeta=P_{\beta}$ we obtain

$$
i\left(\delta, P_{\beta}\right) / \operatorname{ci}\left(P_{\gamma}, P_{\beta}\right) \leq a i\left(P_{\alpha}, P_{\beta}\right) / \operatorname{ci}\left(P_{\gamma}, P_{\beta}\right)=1,
$$

and for $\zeta=P_{\alpha}$ we obtain

$$
i\left(\delta, P_{\alpha}\right) / \operatorname{ci}\left(P_{\gamma}, P_{\alpha}\right) \leq 1 / \operatorname{aci}\left(P_{\gamma}, P_{\alpha}\right)=1 .
$$

Therefore we have $\delta \in L_{c}\left(P_{\gamma}, P_{\beta}, k_{1}\right) \cap L_{a}\left(P_{\alpha}, P_{\beta}, k_{1}\right) \cap L_{c}\left(P_{\gamma}, P_{\alpha}, k_{1}\right)$. Together with Lemma 3.2 and our above remark we conclude that there is a universal constant $D_{2}>0$ such that the distance between $\phi(\alpha, \beta, \gamma)=\delta$ and the image under $\Phi$ of any $\alpha \rightarrow \beta$-splitting sequence, any $\alpha \rightarrow \gamma$-splitting sequence and any $\gamma \rightarrow \beta$-splitting sequence is bounded from above by $D_{2}$.

We use the map $\phi$ to derive the $\delta$-thin triangle condition for triangles whose sides are images under the map $\Phi$ of splitting sequences in $\mathcal{T} T$.

Lemma 3.4. There is a number $D_{3}>0$ with the following property. Let $\alpha, \beta, \gamma \in$ $\mathcal{C}(S)$ and let $a, b, c$ be the image under $\Phi$ of $a \beta \rightarrow \gamma, \gamma \rightarrow \alpha, \alpha \rightarrow \beta$-splitting sequence. Then the $D_{3}$-neighborhood of $a \cup b$ contains $c$.

Proof. Let $\alpha, \beta, \gamma \in \mathcal{C}(S)$ and assume that $d(\beta, \gamma) \leq p$ for some $p>0$. Let $\left(\tau_{i}\right)_{0 \leq i \leq m}$ be an $\alpha \rightarrow \beta$-splitting sequence and let $\left(\eta_{j}\right)_{0 \leq j \leq \ell}$ be an $\alpha \rightarrow \gamma$-splitting sequence; if $D_{1}>0$ is as above then the Hausdorff distance between $\left\{\Phi\left(\tau_{i}\right) \mid 0 \leq i \leq\right.$ $m\}$ and $\left\{\Phi\left(\eta_{j}\right) \mid 0 \leq j \leq \ell\right\}$ is at most $p D_{1}$. Namely, we observed that the Hausdorff distance between the image under $\Phi$ of any two $\alpha \rightarrow \beta$-splitting sequences is bounded from above by $D_{1}$. Moreover, if $d(\beta, \gamma)=1$ then $\beta \cup \gamma \in \mathcal{M C}(S)$ and hence there is an $\alpha \rightarrow \beta$-splitting sequence which also is an $\alpha \rightarrow \gamma$-splitting sequence. Thus the statement of the corollary holds for $p=1$, and the general case follows from a successive application of this fact for the points on a geodesic connecting $\beta$ to $\gamma$.

Now let $\alpha, \beta, \gamma \in \mathcal{C}(S)$ be arbitrary. Let again $\left(\tau_{i}\right)_{0 \leq i \leq m}$ be an $\alpha \rightarrow \beta$-splitting sequence. By the definition of $\phi$ and the choice of the constant $D_{2}>0$ above there is some $i_{0} \leq m$ such that the distance betweeen $\Phi\left(\tau_{i_{0}}\right)$ and $\phi(\alpha, \beta, \gamma)$ is at most $D_{2}$. Let $\left(\eta_{j}\right)_{0 \leq j \leq \ell}$ be any $\alpha \rightarrow \phi(\alpha, \beta, \gamma)$-splitting sequence. By our above consideration, the Hausdorff distance between $\left\{\Phi\left(\tau_{i}\right) \mid 0 \leq i \leq i_{0}\right\}$ and $\left\{\Phi\left(\eta_{j}\right) \mid 0 \leq j \leq \ell\right\}$ is at most $D_{1} D_{2}$. Similarly, let $\left(\zeta_{j}\right)_{0 \leq j \leq n}$ be an $\alpha \rightarrow \gamma$-splitting sequence. Then there is some $j_{0}>0$ such that $d\left(\Phi\left(\zeta_{j_{0}}\right), \phi(\alpha, \beta, \gamma)\right) \leq D_{2}$. By our above argument, the Hausdorff distance between the sets $\left\{\Phi\left(\tau_{i}\right) \mid 0 \leq i \leq i_{0}\right\}$ and $\left\{\Phi\left(\zeta_{j}\right) \mid 0 \leq j \leq j_{0}\right\}$ is at most $2 D_{1} D_{2}$.

On the other hand, the Hausdorff distance between the image under the map $\Phi$ of any $\beta \rightarrow \Phi\left(\tau_{i_{0}}\right)$-splitting sequence and any $\Phi\left(\tau_{i_{0}}\right) \rightarrow \beta$-splitting sequence is at most $D_{1}$. Thus we can apply the above argument to the splitting sequence $\left(\tau_{i}\right)_{i_{0} \leq i \leq m}$ and an $\beta \rightarrow \gamma$-splitting sequence and conclude that $\left\{\Phi\left(\tau_{i}\right) \mid 0 \leq i \leq m\right\}$ is contained in the $3 D_{1} D_{2}$-neighborhood of the union of the image under $\Phi$ of a $\alpha \rightarrow \gamma$-splitting sequence and a $\beta \rightarrow \gamma$-splitting sequence. This shows the lemma. 
We are now ready to give a proof of the hyperbolicity theorem.

Theorem 3.5 ([18, 2]). The curve complex is hyperbolic.

Proof. By Lemma 3.4 it is enough to show the existence of a uniform constant $\kappa>0$ with the following property. For $\alpha, \beta \in \mathcal{C}(S)$ let $\left(\tau_{i}\right)_{0 \leq i \leq m}$ be an $\alpha \rightarrow \beta$-splitting sequence; then for every geodesic $\nu:[0, \ell] \rightarrow \mathcal{C}(S)$ connecting $\alpha$ to $\beta$ the Hausdorffdistance between $\nu[0, \ell]$ and $\left\{\Phi\left(\tau_{i}\right) \mid 0 \leq i \leq m\right\}$ is at most $\kappa$. Namely, if this is the case then for every geodesic triangle with sides $a, b, c$ the side $a$ is contained in the $2 \kappa+D_{3}$-neighborhood of $b \cup c$.

To show our claim, let $c:\left[0,2^{k}\right] \rightarrow \mathcal{C}(S)$ be any simplicial path of length $\ell(c)=2^{k}$ connecting $\alpha$ to $\beta$ and consider the point $c\left(2^{k-1}\right)$. Let $\eta_{1}$ be a $c(0) \rightarrow c\left(2^{k-1}\right)$ splitting sequence and choose similarly a $c\left(2^{k-1}\right) \rightarrow c\left(2^{k}\right)$-splitting sequence $\eta_{2}$. By Lemma 3.4 the $D_{3}$-neighborhood of $\Phi\left(\eta_{1} \cup \eta_{2}\right)$ contains $\left\{\Phi\left(\tau_{i}\right) \mid i \leq m\right\}$. Repeat this construction with the points $c\left(2^{k-2}\right), c\left(3 \cdot 2^{k-2}\right)$ and the splitting sequences $\eta_{1}, \eta_{2}$. Inductively we conclude that the path $\left\{\Phi\left(\tau_{i}\right) \mid 0 \leq i \leq m\right\}$ is contained in the $\left(\log _{2} \ell(c)\right) D_{3}$-neighborhood of a path $\tilde{c}:\left[0,2^{k}\right] \rightarrow \mathcal{C}(S)$ whose restriction to each interval $[m-1, m]\left(m \leq 2^{k}\right)$ is the image under $\Phi$ of a $c(m-1) \rightarrow c(m)$-splitting sequence (compare the discussion in [3]). However, we have $d(c(m-1), c(m))=1$ for all $m$ and therefore $c(m-1) \cup c(m)$ is a multi-curve on $S$ and there is a constant $c(m-1) \rightarrow c(m)$-splitting sequence. This implies that the set $\left\{\Phi\left(\tau_{i}\right) \mid 0 \leq i \leq m\right\}$ is in fact contained in the $\left(\log _{2} \ell(c)\right) D_{3}$-neighborhood of $c\left[0,2^{k}\right]$

Now let $c:[0, k] \rightarrow \mathcal{C}(S)$ be a simplicial geodesic connecting $c(0)=\alpha$ to $c(k)=\beta$ which is parametrized by arc length. Let $i>0$ be such that $\Phi\left(\tau_{i}\right)$ has maximal distance to $c[0, k]$, say that this distance equals $D$. Let $D_{0}>0$ be an upper bound for $d\left(\Phi(\eta), \Phi\left(\eta^{\prime}\right)\right)$ for any train track $\eta \in \mathcal{T} T$ and any train track $\eta^{\prime}$ which can be obtained from $\eta$ by a single split (compare the above discussion and 8 ). Choose some $j>0$ such that $d\left(c(j), \Phi\left(\tau_{i}\right)\right)=D$ and let $i_{1}<i<i_{2}$ be such that $d\left(\Phi\left(\tau_{i}\right), \Phi\left(\tau_{i_{u}}\right)\right) \in\left[2 D, 2 D+D_{0}\right](u=1,2)$. In the case that there is no $i_{1} \in[0, i)$ (or $i_{2} \in(i+1, m]$ ) with $d\left(\Phi\left(\tau_{i}\right), \Phi\left(\tau_{i_{1}}\right)\right) \geq 2 D$ (or $d\left(\Phi\left(\tau_{i}\right), \Phi\left(\tau_{i_{2}}\right)\right) \geq 2 D$ ) we choose $i_{1}=0\left(\right.$ or $\left.i_{2}=m\right)$. Let $j_{u} \in\{0, \ldots, m\}$ be such that $d\left(c\left(j_{u}\right), \Phi\left(\tau_{i_{u}}\right)\right) \leq D(u=1,2)$; such points exist by our choice of $D$. Then the distance between $c\left(j_{1}\right)$ and $c\left(j_{2}\right)$ is at most $6 D+2 D_{0}$. Compose the subarc $c\left[j_{1}, j_{2}\right]$ of $c$ with a geodesic connecting $\Phi\left(\tau_{i_{1}}\right)$ to $c\left(j_{1}\right)$ and a geodesic connecting $c\left(j_{2}\right)$ to $\Phi\left(\tau_{i_{2}}\right)$. We obtain a curve $\nu$ of length at most $8 D+2 D_{0}$. By our above observation, the $\left(\log _{2}\left(8 D+2 D_{0}\right)\right) D_{3}$-neighborhood of this curve contains the set $\left\{\Phi\left(\tau_{s}\right) \mid i_{1} \leq s \leq i_{2}\right\}$. But the distance between $\Phi\left(\tau_{i}\right)$ and our curve $\nu$ equals $D$ by construction and hence we have $D \leq\left(\log _{2}\left(8 D+2 D_{0}\right)\right) D_{3}$. In other words, $D$ is bounded from above by a universal constant $\kappa_{1}>0$ and $\left\{\Phi\left(\tau_{i}\right) \mid 0 \leq i \leq m\right\}$ is contained in the $\kappa_{1}$-neighborhood of the geodesic $c$.

A similar argument also shows that there is a constant $\kappa_{2}>0$ such that the $\kappa_{2}$-neighborhood of $\left\{\Phi\left(\tau_{i}\right) \mid 0 \leq i \leq m\right\}$ contains $c[0, k]$. Namely, by the above consideration, for every $i \leq m$ there is a non-empty subset $A(i)$ of $\{0, \ldots, k\}$ with the property that $d\left(c(s), \Phi\left(\tau_{i}\right)\right) \leq \kappa_{1}$ for all $s \in A(i)$. The cardinality of the sets $A(i)$ is bounded from above by $2 \kappa_{1}$. Now let $j>0$ be such that $c(j)$ has maximal distance to $\left\{\Phi\left(\tau_{i}\right) \mid 0 \leq i \leq m\right\}$, say that this distance equals $D$. We have to show that $D$ is bounded from above by a universal constant, so assume without loss of generality that $D>3 \kappa_{1}$; then $j \notin \cup_{u} A(u)$. Let $j_{1}=\max \{i<j \mid i \in$ $\left.\cup_{u} A(u)\right\}$ and let $j_{2}=\min \left\{i>j \mid i \in \cup_{u} A(u)\right\}$. Choose some $i_{1}, i_{2} \leq m$ such that $d\left(\Phi\left(\tau_{i_{u}}\right), c\left(j_{u}\right)\right) \leq \kappa_{1}(u=1,2)$. Since $c$ is a geodesic parametrized by arc length we have $\left(j_{2}-j_{1}\right) / 2 \geq D-\kappa_{1}$ and moreover the distance between $\Phi\left(\tau_{i_{1}}\right)$ 
and $\Phi\left(\tau_{i_{2}}\right)$ is at least $j_{2}-j_{1}-2 \kappa_{1}$. In particular, there is some $s \in\left(i_{1}, i_{2}\right)$ with $d\left(\Phi\left(\tau_{s}\right),\left\{\Phi\left(\tau_{i_{1}}\right), \Phi\left(\tau_{i_{2}}\right)\right\}\right) \geq\left(j_{2}-j_{1}-2 \kappa_{1}-D_{0}\right) / 2$. Let $t \in[0, k]-\left(j_{1}, j_{2}\right)$ be such that $d\left(c(t), \Phi\left(\tau_{s}\right)\right) \leq \kappa_{1}$. By our above consideration, applied to the splitting sequence $\left(\tau_{i}\right)_{i_{1} \leq i \leq i_{2}}$ and the composition of the $\operatorname{arc} c\left[j_{1}, j_{2}\right]$ with a geodesic segment joining $\Phi\left(\tau_{i_{1}}\right)$ to $c\left(j_{1}\right)$ and a geodesic segment joining $c\left(j_{2}\right)$ to $\Phi\left(\tau_{i_{2}}\right)$, there is a universal constant $\chi>0$ such that the point $c(t)$ is contained in the $\left(\log _{2}\left(j_{2}-j_{1}\right)\right) \chi$ neighborhood of $c\left[j_{1}, j_{2}\right]$. However, the distance between $c(t)$ and the endpoints of the segment $c\left[j_{1}, j_{2}\right]$ is at least $\left(j_{2}-j_{1}-4 \kappa_{1}-D_{0}\right) / 2$ and hence since $c$ is a geodesic, the same estimate holds for the distance between $c(t)$ and $c\left[j_{1}, j_{2}\right]$. This implies that $j_{2}-j_{1}$ and hence $D$ is bounded from above by a universal constant $\kappa_{2}>0$. In other words, the geodesic $c$ is contained in the $\kappa_{2}$-neighborhood of $\left\{\Phi\left(\tau_{i}\right) \mid 0 \leq i \leq m\right\}$. This completes the proof of the theorem.

A curve $c:[0, m] \rightarrow \mathcal{C}(S)$ is called an unparametrized q-quasi-geodesic for some $q>1$ if there is a homeomorphism $\rho:[0, p] \rightarrow[0, m]$ for some $p>0$ such that

$$
d(c(\rho(s)), c(\rho(t))) / q-q \leq|s-t| \leq q d(c(\rho(s)), c(\rho(t)))+q
$$

for all $s, t \in[0, p]$. The following observation is immediate from Theorem 3.5 and its proof.

Corollary 3.6 (19, 8). There is a number $q>0$ such that the image under $\Phi$ of an arbitrary splitting sequence is an unparametrized q-quasi-geodesic.

Proof. By Theorem 3.5 and its proof, there is a universal number $D>0$ with the property that for every splitting sequence $\left(\tau_{i}\right)_{0 \leq i \leq m}$ and every geodesic $c:[0, s] \rightarrow$ $\mathcal{C}(S)$ connecting $c(0)=\Phi\left(\tau_{0}\right)$ to $c(s)=\Phi\left(\tau_{m}\right)$, the Hausdorff distance between the sets $\left\{\Phi\left(\tau_{i}\right) \mid 0 \leq i \leq m\right\}$ and $c[0, s]$ is at most $D$. From this the corollary is immediate.

\section{Geometry of Teichmüller space}

In this section, we relate the geometry of the complex of curves to the geometry of Teichmüller space equipped with the Teichmüller metric. For this we first define a map $\Psi: \mathcal{T}_{g, m} \rightarrow \mathcal{C}(S)$ as follows. By a well know result of Bers (see $[\mathrm{Bu}]$ ) there is a number $\chi>0$ only depending on the topological type of $S$ such that for every complete hyperbolic metric on $S$ of finite volume there is a pants decomposition $P$ for $S$ which consists of simple closed geodesics of length at most $\chi$. Since the distance between any two points $\alpha, \beta \in \mathcal{C}(S)$ is bounded from above by $i(\alpha, \beta)+1$, the collar lemma for hyperbolic surfaces (see [4) implies that the diameter in $\mathcal{C}(S)$ of the set of simple closed geodesics whose length with respect to the fixed metric is at most $\chi$ is bounded from above by a universal constant $D>0$. Define $\Psi: \mathcal{T}_{g, m} \rightarrow$ $\mathcal{C}(S)$ by assigning to a hyperbolic metric $h$ on $S$ a simple closed curve $\Psi(h)$ whose $h$-length is at most $\chi$. Then for any two maps $\Psi, \Psi^{\prime}$ with this property and every $h \in \mathcal{T}_{g, m}$ the distance in $\mathcal{C}(S)$ between $\Psi(h)$ and $\Psi^{\prime}(h)$ is at most $D$. Moreover, the map $\Psi$ is coarsely equivariant with respect to the action of the mapping class group $\mathcal{M}_{g, m}$ on $\mathcal{T}_{g, m}$ and $\mathcal{C}(S)$ : For every $h \in \mathcal{T}_{g, m}$ and every $\phi \in \mathcal{M}_{g, m}$ we have $d(\Psi(\phi(h)), \phi(\Psi(h))) \leq D$.

The following result is due to Masur and Minsky (Theorem 2.6 and Theorem 2.3 of [18]); a refined quantitative version can be obtained from the results of Rafi [24]. 
Theorem $4.1([18])$. There is a number $\tilde{q}>0$ with the following property. Let $\gamma:(-\infty, \infty) \rightarrow \mathcal{T}_{g, m}$ be any Teichmüller geodesic; then the assignment $t \rightarrow \Psi(\gamma(t))$ is an unparametrized $\tilde{q}$-quasi-geodesic in $\mathcal{C}(S)$.

Proof. Every Teichmüller geodesic is determined by a pair of projective measured geodesic laminations which jointly fill up $S$. The projective measured geodesic laminations defined by simple closed geodesics are dense in $\mathcal{P} \mathcal{M L}$ and hence the Teichmüller geodesics determined by a pair of curves $\alpha, \beta \in \mathcal{C}(S)$ which jointly fill up $S$ are dense in the set of all Teichmüller geodesics equipped with the compact open topology. Since the map $\Psi$ is coarsely Lipschitz it is therefore enough to assume that our geodesic is of this form.

Thus let $\alpha, \beta \in \mathcal{C}(S)$ with $d(\alpha, \beta) \geq 3$ and for $a>0$ let $q(a \alpha, \beta)$ be the holomorphic quadratic differential of area one on a Riemann surface $h \in \mathcal{T}_{g, m}$ whose horizontal foliation corresponds to the measured lamination $a \alpha$ and whose vertical foliations corresponds to a multiple of $\beta$. A geodesic segment for the differential $q=q(a \alpha, \beta)$ is a path in $S$ not containing any singularities in its interior and which is a geodesic in the local euclidean structure defined by $q$. A closed geodesic is composed of a finite number of such geodesic segments which meet at singular points of $q$ and make an angle at least $\pi$ on either side. Every essential simple closed curve on $S$ can be realized by a simple closed geodesic with respect to $q$, and the length of such a geodesic $\eta$ is the infimum of the $q$-lengths of any curve freely homotopic to $\eta$ (compare [24] for a detailed discussion of the technical difficulties caused by the punctures of $S$ ).

The horizontal length $h_{q}(\eta)$ (or vertical length $v_{q}(\eta)$ ) of a simple closed curve $\eta$ on $S$ is defined as follows. Every tangent vector $X$ of $S$ at a regular point for $q$ can uniquely be decomposed as $X=\operatorname{hor}(X)+\operatorname{vert}(X)$ where hor $(X)$ is tangent to the horizontal foliation and $\operatorname{vert}(X)$ is tangent to the vertical foliation. Then the horizontal length (or vertical length) of $\eta$ is the infimum of the integrals $\int_{\zeta}\left|\operatorname{hor}\left(\zeta^{\prime}\right)\right|$ (or $\int_{\zeta}\left|\operatorname{vert}\left(\zeta^{\prime}\right)\right|$ ) where $\zeta$ ranges through all simple closed curves which are freely homotopic to $\eta$. The curve $\eta$ is called balanced with respect to $q$ if $h_{q}(\eta)=v_{q}(\eta)$. For every simple closed curve $\eta \in \mathcal{C}(S)$ with $i(\eta, \alpha)>0, i(\eta, \beta)>0$ there is a unique $a>0$ such that $\eta$ is balanced with respect to the differential $q(a \alpha, \beta)$.

Theorem 6.1 of [24] shows that there is a universal constant $\kappa>0$ such that the following holds. For $\eta \in \mathcal{S}(S)$ define $\ell(\eta)$ to be the infimum of the hyperbolic lengths of $\eta$ along the Teichmüller geodesic in $\mathcal{T}_{g, m}$ determined by the quadratic differentials $q(a \alpha, \beta)$. If $i(\eta, \alpha)>0, i(\eta, \beta)>0$ and if $a>0$ is such that $\eta$ is balanced with respect to the quadratic differential $q(a \alpha, \beta)$, then the length of $\eta$ with respect to the hyperbolic metric determined by the complex structure $q(a \alpha, \beta)$ is not bigger than $\kappa \ell(\eta)$. Moreover, by Theorem 5.6 of [24], for every simple closed curve $\zeta$ on $S$ which intersects $\eta$ transversely we have

$$
i(\eta, \zeta) \leq K \max \{\operatorname{ai}(\zeta, \alpha), i(\zeta, \beta) / a i(\alpha, \beta)\} .
$$

In other words, every essential simple closed curve $\eta$ on $S$ which has an essential intersection with both $\alpha, \beta$ and whose length with respect to the hyperbolic metric determined by $q(a \alpha, \beta)$ is at most $\chi$ is contained in a uniformly bounded neighborhood of the set $L_{a}(\alpha, \beta, K)$ defined in Section 3. By the considerations in Section 3, this just means that the image under $\Psi$ of the Teichmüller geodesic determined by $\alpha$ and $\beta$ is a uniform unparametrized quasi-geodesic in the complex of curves. 
There are also geodesics in Teichmüller space which are mapped by $\Psi$ to parametrized quasi-geodesics in $\mathcal{C}(S)$. For their characterization, denote for $\epsilon>0$ by $\mathcal{T}_{g, m}^{\epsilon}$ the subset of Teichmüller space consisting of all hyperbolic metrics for which the length of the shortest closed geodesic is at least $\epsilon$. The set $\mathcal{T}_{g, m}^{\epsilon}$ is invariant under the action of the mapping class group and projects to a compact subset of moduli space. Moreover, every compact subset of moduli space is contained in the projection of $\mathcal{T}_{g, m}^{\epsilon}$ for some $\epsilon>0$.

Teichmüller geodesics which project to a compact subset of moduli space relate the geometry of Teichmüller space to the geometry of the complex of curves. We have.

Proposition $4.2([9])$. The image under $\Psi$ of a Teichmüller geodesic $\gamma: \mathbb{R} \rightarrow \mathcal{T}_{g, m}$ is a parametrized quasi-geodesic in $\mathcal{C}(S)$ if and only if there is some $\epsilon>0$ such that $\gamma(\mathbb{R}) \subset \mathcal{T}_{g, m}^{\epsilon}$.

Minsky [20] discovered earlier that the Teichmüller metric near a cobounded geodesic line has properties similar to properties of a hyperbolic geodesic metric space. Namely, for a geodesic $\gamma: \mathbb{R} \rightarrow \mathcal{T}_{g, m}^{\epsilon}$ the map which associates to a point $h \in \mathcal{T}_{g, m}$ a point on $\gamma(\mathbb{R})$ which minimizes the Teichmüller distance is coarsely Lipschitz and contracts distances similar to the closest point projection from a $\delta$-hyperbolic geodesic metric space to a bi-infinite geodesic.

A hyperbolic geodesic metric space $X$ admits a Gromov boundary which is defined as follows. Fix a point $p \in X$ and for two points $x, y \in X$ define the Gromov product $(x, y)_{p}=\frac{1}{2}(d(x, p)+d(y, p)-d(x, y))$. Call a sequence $\left(x_{i}\right) \subset X$ admissible if $\left(x_{i}, x_{j}\right)_{p} \rightarrow \infty(i, j \rightarrow \infty)$. We define two admissible sequences $\left(x_{i}\right),\left(y_{i}\right) \subset X$ to be equivalent if $\left(x_{i}, y_{i}\right)_{p} \rightarrow \infty$. Since $X$ is hyperbolic, this defines indeed an equivalence relation (see [3]). The Gromov boundary $\partial X$ of $X$ is the set of equivalence classes of admissible sequences $\left(x_{i}\right) \subset X$. It carries a natural Hausdorff topology. For the complex of curves, the Gromov boundary was determined by Klarreich 14 (see also [8]).

For the formulation of Klarreich's result, we say that a minimal geodesic lamination $\lambda$ fills up $S$ if every simple closed geodesic on $S$ intersects $\lambda$ transversely, i.e. if every complementary component of $\lambda$ is an ideal polygon or a once punctured ideal polygon with geodesic boundary [5]. For any minimal geodesic lamination $\lambda$ which fills up $S$, the number of geodesic laminations $\mu$ which contain $\lambda$ as a sublamination is bounded by a universal constant only depending on the topological type of the surface $S$. Namely, each such lamination $\mu$ can be obtained from $\lambda$ by successively subdividing complementary components $P$ of $\lambda$ which are different from an ideal triangle or a once punctured monogon by adding a simple geodesic line which either connects two non-adjacent cusps or goes around a puncture. Note that every leaf of $\mu$ which is not contained in $\lambda$ is necessarily isolated in $\mu$.

Recall that the space $\mathcal{L}$ of geodesic laminations on $S$ equipped with the restriction of the Hausdorff topology for compact subsets of $S$ is compact and metrizable. It contains the set $\mathcal{B}$ of minimal geodesic laminations which fill up $S$ as a subset which is neither closed nor dense. We define on $\mathcal{B}$ a new topology which is weaker than the restriction of the Hausdorff topology as follows. Say that a sequence $\left(\lambda_{i}\right) \subset \mathcal{L}$ converges in the coarse Hausdorff topology to a minimal geodesic lamination $\mu$ which fills up $S$ if every accumulation point of $\left(\lambda_{i}\right)$ with respect to the Hausdorff topology contains $\mu$ as a sublamination. Define a subset $A$ of $\mathcal{B}$ to be closed if and only if for every sequence $\left(\lambda_{i}\right) \subset A$ which converges in the coarse Hausdorff 
topology to a lamination $\lambda \in \mathcal{B}$ we have $\lambda \in A$. We call the resulting topology on $\mathcal{B}$ the coarse Hausdorff topology. Note that every subset of $A$ of $\mathcal{B}$ which is closed with respect to the coarse Hausdorff topology is the intersection with $\mathcal{B}$ of a closed subset of $\mathcal{L}$ with respect to the usual Hausdorff topology. However, the space $\mathcal{B}$ is not locally compact. Using this terminology, Klarreich's result 14 can be formulated as follows.

Theorem 4.3 (14, 8). (1) There is a natural homeomorphism $\Lambda$ of $\mathcal{B}$ equipped with the coarse Hausdorff topology onto the Gromov boundary $\partial \mathcal{C}(S)$ of the complex of curves $\mathcal{C}(S)$ for $S$.

(2) For $\mu \in \mathcal{B}$ a sequence $\left(c_{i}\right) \subset \mathcal{C}(S)$ is admissible and defines the point $\Lambda(\mu) \in \partial \mathcal{C}(S)$ if and only if $\left(c_{i}\right)$ converges in the coarse Hausdorff topology to $\mu$.

Recall that every Teichmüller geodesic in $\mathcal{T}_{g, m}$ is uniquely determined by a pair of projective measured laminations which jointly fill up $S$. The following corollary is immediate from Theorem 4.1 and Theorem 4.2.

Corollary 4.4. Let $\lambda, \mu \in \mathcal{P} \mathcal{M L}$ be such that their supports are minimal and fill up $S$. Then the image under $\Psi$ of the unique Teichmüller geodesic in $\mathcal{T}_{g, m}$ determined by $\lambda$ and $\mu$ is a biinfinite unparametrized $\tilde{q}$-quasigeodesic in $\mathcal{C}(S)$, and every biinfinite unparametrized $\tilde{q}$-quasi-geodesic in $\mathcal{C}(S)$ is contained in a uniformly bounded neighborhood of a curve of this form.

Our above discussion also gives informations on images under $\Psi$ of a convergent sequence of geodesic lines in Teichmüller space. Namely, if $(\gamma)_{i}$ is such a sequence of Teichmüller geodesic lines converging to a Teichmüller geodesic which is determined by a pair of projective measured laminations $(\alpha, \beta)$ so that the support of $\alpha$ does not fill up $S$ then there is a curve $\zeta \in \mathcal{C}(S)$, a number $m>0$ and there is a sequence $j(i) \rightarrow \infty$ such that $d\left(\Psi\left(\gamma_{i}[0, j(i)]\right), \zeta\right) \leq m$.

On the other hand, there are Teichmüller geodesics whose images under $\Psi$ are unparametrized quasi-geodesics of infinite diameter and which are not parametrized quasi-geodesics. Namely, let $\lambda \in \mathcal{P} \mathcal{M L}$ be a projective measured geodesic lamination whose support $\lambda_{0}$ is minimal and fills up $S$ but is not uniquely ergodic. This means that the dimension of the space of transverse measures supported in $\lambda_{0}$ is at least 2. Let $\gamma:[0, \infty) \rightarrow \mathcal{T}_{g, m}$ be a Teichmüller geodesic ray determined by a quadratic differential whose horizontal foliation corresponds to $\lambda$. By a result of Masur [16], $\gamma[0, \infty)$ does not project to a compact subset of moduli space. On the other hand, since $\lambda_{0}$ is minimal and fills up $S$ the points $\gamma(t)$ converge as $t \rightarrow \infty$ to $\lambda$ viewed as a point in the Thurston boundary of the Thurston compactification of Teichmüller space [17] (compare also [6] for the construction of the Thurston compactification). By the definition of the map $\Psi$, the projective measured geodesic laminations defined by the curves $\Psi \gamma(t)$ then converge as $t \rightarrow \infty$ to $\lambda$ and therefore the curves $\Psi \gamma(t)$ converge in the coarse Hausdorff topology to $\lambda_{0}$. By Theorem 4.3, this implies that the diameter of $\Psi \gamma[0, \infty)$ is infinite.

\section{REFERENCES}

[1] F. Bonahon, Geodesic laminations on surfaces, in Laminations and foliations in dynamics, geometry and topology, (Stony Brook, NY, 1998), 1-37, Contemp. Math., 269, Amer. Math. Soc., Providence, RI, 2001.

[2] B. Bowditch, Intersection numbers and the hyperbolicity of the curve complex, preprint 2002. 
[3] M. Bridson, A. Haefliger, Metric spaces of non-positive curvature, Springer Grundlehren 319, Springer, Berlin 1999.

[4] P. Buser, Geometry and spectra of compact Riemann surfaces, Birkhäuser, Boston 1992.

[5] R. Canary, D. Epstein, P. Green, Notes on notes of Thurston, in Analytical and geometric aspects of hyperbolic space, edited by D. Epstein, London Math. Soc. Lecture Notes 111, Cambridge University Press, Cambridge 1987.

[6] A. Fathi, F. Laudenbach, V. Poénaru, Travaux de Thurston sur les surfaces, Astérisque 66-67, Soc. Math. Fr. 1991/1979.

[7] U. Hamenstädt, Train tracks and mapping class groups I, preprint 2004, available at http://www.math.uni-bonn.de/ ursula

[8] U. Hamenstädt, Train tracks and the Gromov boundary of the complex of curves, math.GT/0409611 to appear in Proc. Newton Inst, Cambridge University Press.

[9] U. Hamenstädt, Subgroups of mapping class groups and the complex of curves, preprint 2005, available at http://www.math.uni-bonn.de/ ursula

[10] W. J. Harvey, Boundary structure of the modular group, in Riemann Surfaces and Related topics: Proceedings of the 1978 Stony Brook Conference, edited by I. Kra and B. Maskit, Ann. Math. Stud. 97, Princeton, 1981.

[11] Y. Imayoshi, M. Taniguchi, An introduction to Teichmüller spaces, Springer, Tokyo 1992.

[12] N. V. Ivanov, Mapping class groups, Chapter 12 in Handbook of geometric topology, edited by R. J. Daverman and R. B. Sher, Elsevier Science 2002, 523-633.

[13] S. Kerckhoff, Lines of minima in Teichmüller space, Duke Math. J. 65 (1992), 187-213.

[14] E. Klarreich, The boundary at infinity of the curve complex and the relative Teichmüller space, preprint 1999.

[15] G. Levitt, Foliations and laminations on hyperbolic surfaces, Topology 22 (1983), 119-135.

[16] H. Masur, Interval exchange transformations and measured foliations, Ann. Math. 155 (1982), 169-200.

[17] H. Masur, Two boundaries of Teichmüller space, Duke Math. J. 49 (1982), 183-190.

[18] H. Masur, Y. Minsky, Geometry of the complex of curves I: Hyperbolicity, Invent. Math. 138 (1999), 103-149.

[19] H. Masur, Y. Minsky, Quasiconvexity in the curve complex, preprint 2003.

[20] Y. Minsky, Quasi-projections in Teichmüller space, J. Reine Angew. Math. 473 (1996), 121136.

[21] L. Mosher, Train track expansions of measured foliations, unpublished manuscript.

[22] J. P. Otal, Le Théorème d'hyperbolisation pour les variétés fibrées de dimension 3, Astérisque 235, Soc. Math. Fr. 1996.

[23] R. Penner with J. Harer, Combinatorics of train tracks, Ann. Math. Studies 125, Princeton University Press, Princeton 1992.

[24] K. Rafi, A characterization of short curves of a Teichmüller geodesic, Geom. Top. 9 (2005), 179-202.

[25] K. Strebel, Quadratic differentials, Ergebnisse der Mathematik und ihrer Grenzgebiete (3) 5, Springer, Berlin 1984 